\title{
Underwater discovery of Roman surgical equipment
}

\author{
Christopher Edge, David Gibbins
}

Oxford Glycobiology Unit, University of Oxford, Oxford OX1 3QU Christopher Edge, PHD, research fellow

\section{Department of}

Archaeology, University of Cambridge, Cambridge CB2 3DZ

David Gibbins, MA, research scholar, Corpus Christi College

Correspondence to: D Gibbins.
Ancient shipwrecks can yield much information about contemporary culture, such as the food eaten, clothes worn, and domestic equipment used. Off the south east coast of Sicily, $3 \mathrm{~km}$ from the ancient city of Syracuse, lie the remains of a Roman shipwreck known simply as "Plemmiro B." Pottery amphoras, the "jerrycans" of antiquity, are scattered on the seabed at a depth of $30 \mathrm{~m}$. These and objects from the kitchen stores - terracotta lamps, glass vessels, and cooking pots-have been dated and show that the ship foundered around $\mathrm{AD} 200$. She was carrying a cargo of fish paste, olive oil, and iron bars when she sank, probably in one of the fierce storms that are common off the Sicilian coast.

Investigation of the site of the wreck in 1983-7 under the guidance of one of us (DG) led to a unique find. Careful sifting of the black sediment at the bottom of a gully uncovered a small bronze object. At first we thought that it was part of the ship's equipment, though we were uncertain of its purpose. Further research, however, showed that it was a bronze scalpel handle, the first recorded specimen found from an ancient shipwreck. The long slender point was a blunt dissector, but the crescent shaped iron scalpel blade that would have been attached to the other end had corroded away: iron rarely survives in archaeological sites, and few extant examples of such blades are known.

Subsequent excavation uncovered two more scalpel handles of identical manufacture to the first (although one was without the blunt dissector) and a long wooden shaft, which may have been a "bandaging stick" for winding tourniquets. The scalpel without the blunt dissector had been combined with a second instrument made of iron, the remains of which were preserved in a hole at the end of the handle. This was probably a solid cataract needle - a rare combination with a scalpel. All three handles showed metallic residue from the iron scalpel blades as well as the remains of solder or iron wire used to bind them securely in place.

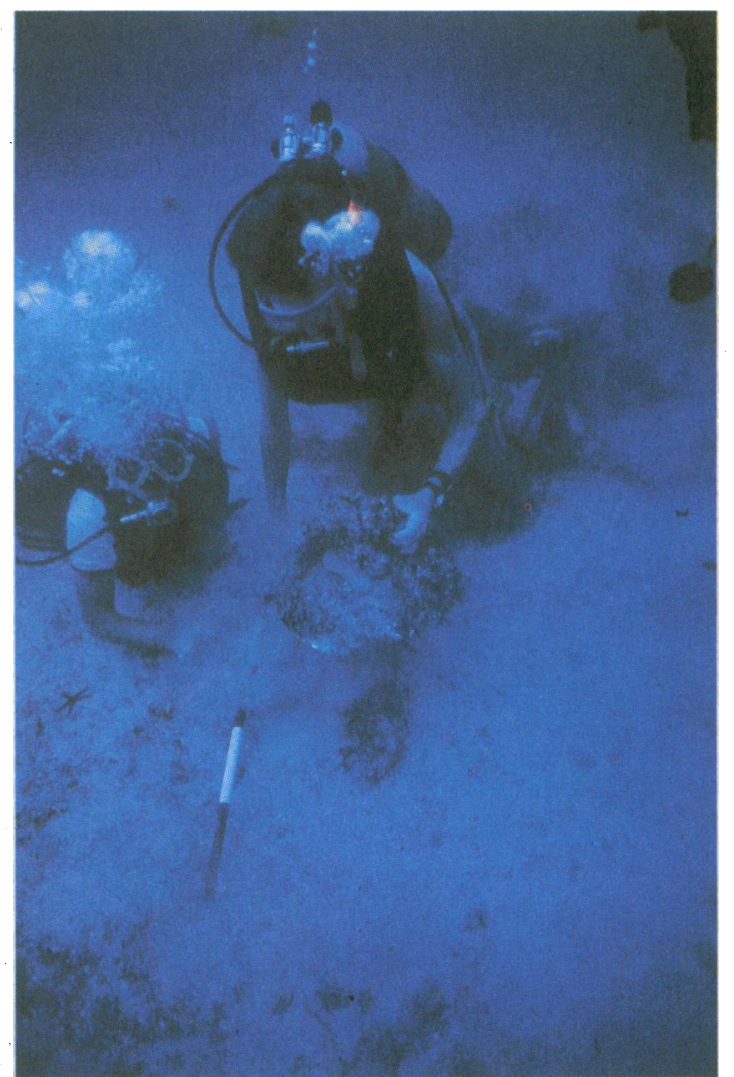

Excavation at a depth of $50 \mathrm{~m}$ of amphora, later shown to be Greek and nearly 3000 years old

The manufacture of handles and mending of broken blades were probably carried out by a smith or cutler or perhaps a specialist instrument maker. These objects display both elegance of form and a refined understanding of the practical requirements of their use. They represent the peak of Roman bronze work, and the manufacturer of such precision equipment probably had the status of a modern jeweller or clock maker. In Ostia, the port of Rome, a terracotta relief from the second century shows a specialist smith with a small boxed set of scalpels by his side.

The carefully designed rolls and perforations at the end of such handles originally were thought not only to hold the blade but also to be a feature designed to help the interchanging of blades so that a surgeon's kit, or instrumentarium, might comprise several handles with a range of blades and other attachments. The arrangement of slots at the end of the handle would allow a broken blade to be replaced without the handle having to be discarded, but there is now some dispute over whether blades would have been changed regularly. The principal evidence against this theory is the large number of handles found in complete instrumentariums - for instance, one from a surgeon's grave in Bingen, Germany, contained 13 such handles. Possibly the rolls at the ends of the handles were largely decorative. 
tion for the removal of dermoid cysts: "Then as soon as the white and tight coat is seen, it is to be separated from the skin and flesh by the handle of the scalpel and turned out together with its contents." His account of cataract removal, for which a handle with a needle was used, is a graphic illustration of the extraordinary refinements of Roman eye surgery: "Thereupon a needle is to be taken pointed enough to penetrate, yet not too fine; and this is to be inserted straight through the two outer tunics at a spot intermediate between the pupil of the eye and the angle adjacent to the temple, away from the middle of the cataract, in such a way that no vein is wounded."

\section{Travelling doctor}

Probably the finds from the Plemmirio site are part of a complete instrumentarium and other items from this kit, which could range from bone mallets to metal urinary catheters, remain buried at the site. If this is the case the finds are probably the belongings of a surgeon or an oculist rather than odds and ends from

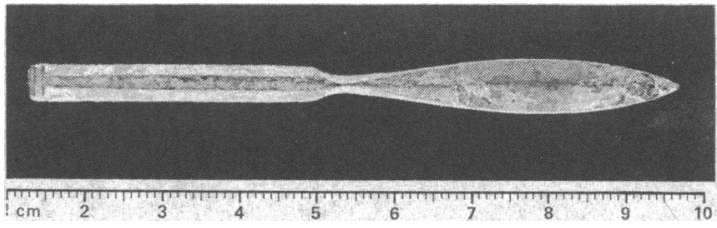

Bronze scalpel handle from wreck of Roman cargo ship

the ship's first aid box. We suggest that the doctor was travelling on board the ship as a passenger. This theory is supported by the unique nature of these finds among the 800 or so ancient wrecks that have been investigated in the Mediterranean and the consequent lack of evidence for doctors in ships' crews. These finds are not only of archaeological interest but also provide an exciting contact with someone who, although dead for almost two milleniums, had skills and concerns with which we can identify.

1 Celsus AAC. De medecina. London: Heineman, 1935-38. (Translated by W G Spencer.)

\section{From Aretaeus to Crosby: a history of coeliac disease}

William F Paveley

The first description of coeliac disease was probably given in the second century $\mathrm{AD}$ by Aretaeus the Cappadocian (fig 1).

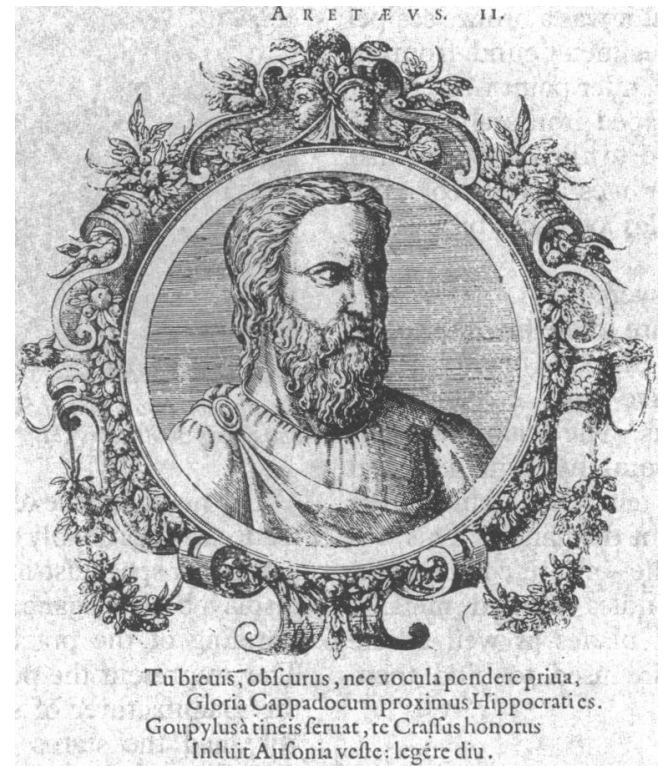

FIG I-Aretaeus the Cappadocian by $\mathcal{F}$ Sambucus, 1531-84

Aretaeus's works were translated by Francis Adams and published by the Sydenham Society in $1856 .^{.}$I paraphrase from this translation.

Derby DE7 3LG

William F Paveley, MB, general practitioner

Correspondence to:

"Heathend," 22 Station

Road, Borrowash, Derby DE7 32G.

The article is based on a presidential address given to Derby Medical Society in October 1983.
The stomach being the digestive organ, labours in digestion when diarrhoea seizes the patient. If this diarrhoea does not proceed from a slight cause of only one or two days' duration, and if, in addition, the patient's general system be debilitated by atrophy of the body, the Coeliac Disease of a chronic nature is formed.

\section{Aretaeus thought that the disease was due to:}

Atony of the heat which digests, and refrigeration of the stomach, when the food indeed is dissolved in the heat but the heat does not digest it, nor convert it into its proper chyme, but leaves its work half-finished. The food then being deprived of this operation, is changed to a state which is bad in colour, smell and consistence. For its colour is white and without bile, it has an offensive smell and is flatulent; it is liquid and wants consistence from not being completely elaborated, and from no part of the digestive process having been properly done except the commencement

\section{He describes the patient as being:}

Emaciated and atrophied, pale, feeble and incapable of performing any of his accustomed works, but if he attempts to walk, the limbs fail, the veins in the temples are prominent, for owing to wasting the temples are hollow, but also all over the body the veins are enlarged. Not only does the disease not digest properly but it does not distribute that portion in which the digestion has commenced. It appears to me, therefore, to be an affection not only of digestion but also of distribution.

Aretaeus, however, thought that this illness affected only adult patients-more common among the aged and particularly in women. Treatment consisted of rest and fasting. Pepsis was promoted by the prevention of chilling and restoration of heat. Changes in the mode of living, exercise, and massage were recommended. If these failed powerful emetics and purges were to be tried. He mentioned diet in passing. He did prescribe "drinks taken before meals, for otherwise bread is very little conducive to trim vigour." I am not suggesting that this brief reference to the ill effects of bread anticipated the findings of later workers in the unravelling of the gluten enigma. As Dowd and Walker-Smith said, "Aretaeus was wise but scarcely so prescient."

It was not until October 1887-1700 years laterthat Dr Samuel Jones Gee again drew attention to coeliac disease (fig 2). The title of his lecture was "On the Coeliac Affection." "This is the title used by Aretaeus and it has been suggested that the choice was an oblique compliment to the earlier Greek physician. Gee was the first person to note that the disease affected all ages:

There is a kind of chronic indigestion which is met with in persons of all ages, yet especially apt to affect children between one and five years old. Signs of the disease are yielded 